\title{
Best Response Analysis in Two Person Quantum Games
}

\author{
Azharuddin Shaik, Aden Ahmed \\ Department of Mathematics, Texas A \& M University-Kingsville, Kingsville, Texas, USA \\ Email: azharms2012@gmail.com, aden.ahmed@tamuk.edu
}

Received 25 May 2014; revised 25 June 2014; accepted 2 July 2014

Copyright (C) 2014 by author and Scientific Research Publishing Inc.

This work is licensed under the Creative Commons Attribution International License (CC BY).

http://creativecommons.org/licenses/by/4.0/

c) (i) Open Access

\begin{abstract}
In this paper, we find particular use for a maximally entangled initial state that produces a quantized version of two player two strategy games. When applied to a variant of the well-known game of Chicken, our construction shows the existence of new Nash equilibria with the players receiving better payoffs than those found in literature.
\end{abstract}

\section{Keywords}

Quantum Games, Nash Equilibrium, Quaternions, Best Response Analysis, Game Extensions

\section{Introduction}

We consider an arbitrary two player, two strategy game whose payoff function is given in Table 1 below.

By a pure classical game $G$, we mean the quadruple $G=\left(S_{1}, S_{2}, P_{1}, P_{2}\right)$, where $S_{1}=\left\{s_{1}, s_{2}\right\}$ and $S_{2}=\left\{t_{1}, t_{2}\right\}$ are the pure strategy spaces of Player 1 and Player 2, respectively, and

$$
P_{1}: S_{1} \times S_{2} \rightarrow\left\{x_{i} \mid i=0,1,2,3\right\}
$$

and

Table 1. Generic two player, two strategy game.

\begin{tabular}{cccc}
\hline & \multicolumn{2}{c}{ Player II } \\
\cline { 2 - 4 } Player I & $\mathrm{t}_{1}$ & $\mathrm{t}_{2}$ \\
\cline { 2 - 4 } & $\mathrm{s}_{1}$ & $\left(\mathrm{x}_{0}, \mathrm{y}_{0}\right)$ & $\left(\mathrm{x}_{3}, \mathrm{y}_{3}\right)$ \\
& $\mathrm{s}_{2}$ & $\left(\mathrm{x}_{2}, \mathrm{y}_{2}\right)$ & $\left(\mathrm{x}_{1}, \mathrm{y}_{1}\right)$ \\
\hline
\end{tabular}




$$
P_{2}: S_{1} \times S_{2} \rightarrow\left\{y_{i} \mid i=0,1,2,3\right\}
$$

are the payoff functions for Player 1 and Player 2, respectively.

Note that in the game $G$ the players' pure strategies are discrete. Suppose now that the players are allowed to randomize their pure strategies, i.e. they can employ real convex combinations of their pure strategies, that is, they can use mixed strategies. For example, Player 1 could observe a fair coin and decide to play $s_{1}$ if it falls Heads and $s_{2}$ if it falls Tails. We will denote the set of probability distributions over the set $S_{i}$ by $\Delta\left(S_{i}\right)$ and observe that, for a player, selecting a mixed strategy is equivalent to choosing a number in the unit interval $[0,1]$. More specifically, the mixed strategy spaces of the players are

$$
\Delta\left(S_{1}\right)=\left\{p s_{1}+(1-p) s_{2} \mid 0 \leq p \leq 1\right\} \equiv[0,1]
$$

and

$$
\Delta\left(S_{2}\right)=\left\{q t_{1}+(1-q) t_{2} \mid 0 \leq q \leq 1\right\} \equiv[0,1],
$$

respectively. Given a profile $(p, q)$ of probability distributions over the $S_{i}$ 's, Player $i$ obtains an expected outcome given by a probability distribution over the outcomes of $G$, that is an element of $\Delta\left(\operatorname{Im} P_{i}\right)$, the set of probability distributions over the image of $P_{i}$. Now the game $G$ is extended to a new, larger game $G^{\text {mix }}$, the mixed classical game associated to $G$. By a mixed classical game $G^{\text {mix }}$, we mean the quadruple $G^{\text {mix }}=\left(\Delta\left(S_{1}\right), \Delta\left(S_{2}\right) ; \in_{1}, \in_{2}\right)$, where

$$
\epsilon_{i}:[0,1] \times[0,1] \rightarrow \Delta\left(\operatorname{Im} P_{i}\right)
$$

is Player $i$ 's expected payoff function. More explicitly, if Player 1 uses his pure strategy $s_{1}$ with probability $p$ and Player 2 uses her pure strategy $t_{1}$ with probability $q$, then the expected outcome to Player $i$ is given by

$$
\epsilon_{i}(p, q)=p q P_{i}\left(s_{1}, t_{1}\right)+p(1-q) P_{i}\left(s_{1}, t_{2}\right)+(1-p) q P_{i}\left(s_{2}, t_{1}\right)+(1-p)(1-q) P_{i}\left(s_{2}, t_{2}\right)
$$

or in matrix form

$$
\epsilon_{i}(p, q)=\left(\begin{array}{ll}
p & 1-p
\end{array}\right)\left(\begin{array}{ll}
P_{i}\left(s_{1}, t_{1}\right) & P_{i}\left(s_{1}, t_{2}\right) \\
P_{i}\left(s_{2}, t_{1}\right) & P_{i}\left(s_{2}, t_{2}\right)
\end{array}\right)\left(\begin{array}{c}
q \\
1-q
\end{array}\right)
$$

Note that the map $\epsilon_{i}$ is not necessarily onto. For example, an easy exercise shows that the element

$$
\frac{1}{2} P_{i}\left(s_{1}, t_{1}\right)+\frac{1}{2} P_{i}\left(s_{2}, t_{2}\right)
$$

of $\Delta\left(\operatorname{Im} P_{i}\right)$ is not realizable by any choice of $p$ and $q$.

This observation motivates the quest for a higher randomization apparatus: quantum superposition followed by quantum measurement. More specifically, let us assume that $H$ is a finite-dimensional complex vector space, and that we have a finite set $X$ which is in one-to-one correspondence with an orthogonal basis $B$ of $H$. By a quantum superposition of $X$ with respect to the basis $B$ we mean a complex projective linear combination of elements of $X$; that is, a representative of an equivalence class of complex linear combinations where the equivalence between combinations is given by non-zero scalar multiplication. Quantum mechanics calls this scalar a phase. When the context is clear as to the basis to which the set $X$ is identified, denote the set of quantum superpositions for $X$ as $Q S(X)$.

As the underlying space of complex linear combinations is a Hilbert space, we can assign a length to each linear combination and, up to phase, always represent a projective linear combination by a complex linear combination of Length 1 . This process is called normalization and is frequently useful.

For each quantum superposition of $X$ we can obtain a probability distribution over $X$ by assigning to each component the ratio of the square of the length of its coefficient to the square of the length of the combination. For example, the probability distribution produced from the quantum superposition $\alpha x+\beta y$ is just

$$
\frac{|\alpha|^{2}}{|\alpha|^{2}+|\beta|^{2}} x+\frac{|\beta|^{2}}{|\alpha|^{2}+|\beta|^{2}} y
$$

This process is called a quantum measurement with respect to $X$, and note that geometrically quantum mea- 
surement is defined by projecting a normalized quantum superposition onto the various elements of the normalized basis $B$.

Now assume that our game $G$ is played under mediated quantum communication a la Eisert, Lewenstein, and Wilkins (EWL) [1] That is, players have a referee mediate their game and the communication of their strategic choices over quantum channels. When there are two strategic choices for each player in the classical game, players and the referee communicate over quantum channels via qubits, a two pure state quantum system with a fixed observational basis. This observational basis is given in the so-called Dirac notation by $|0\rangle$ and $|1\rangle$. This basis also induces an observational basis of the space of the joint states of the players' qubits denoted in the Dirac notation by $|00\rangle,|01\rangle,|10\rangle,|11\rangle$. First, the referee prepares two qubits in the initial state $\psi$, an element of the Hilbert space $\mathrm{H}_{4}$ and of the form

$$
\psi=\frac{|00\rangle+i|11\rangle}{\sqrt{2}}
$$

The referee sends each player one of the qubits. Players then send back their individual qubits in the other state (Flipped) or in the original state (Un-Flipped) to indicate the choice of their second or first classical pure strategy, respectively. The returned qubits are examined by the referee who then makes the appropriate payoffs. So, under this description, we can think of the game $G$ as a two-player two-strategy game in which both players have the same set of pure strategies, namely \{No Flip, Flip\}. The actions No Flip and Flip are elements of the set of special unitary matrices, $S U(2)$, i.e.

$$
S U(2)=\left\{\left(\begin{array}{cc}
x & y \\
-\bar{y} & \bar{x}
\end{array}\right) \mid x, y \in \mathbf{C} \text { and }|x|^{2}+|y|^{2}=1\right\}
$$

More specifically, let the actions No Flip and Flip be represented by the $S U(2)$ matrices

$$
N=\left(\begin{array}{ll}
1 & 0 \\
0 & 1
\end{array}\right) \text { and } F=\left(\begin{array}{cc}
0 & \eta \\
-\bar{\eta} & 0
\end{array}\right)
$$

respectively, where $\eta$ is a unit complex number to be determined shortly. So, when the players are restricted to use only classical pure strategies $N$ and $F$, one can redefine the game $G$ as

$$
G=\left\{\psi ;\{N, F\},\{N, F\} ; P_{1}, P_{2}\right\}
$$

When the players are allowed to randomize their pure strategies, i.e. play real convex combinations of $N$ and $F$ or mixed strategies, we can redefine the game $G^{\text {mix }}$ as

$$
G^{\mathrm{mix}}=\left\{\psi ; \Delta(\{N, F\}), \Delta(\{N, F\}) ; \in_{1}, \in_{2}\right\}
$$

Note that $G^{\text {mix }}$ is an extension of $G$ as the restriction of the expected function $\epsilon_{i}$ to the set $S_{1} \times S_{2}$ is the payoff function $P_{i}$, that is, $\epsilon_{i \mid S_{1} \times S_{2}}=P_{i}$.

Next, we study the situation when the players are free to operate on their respective qubits via general elements of $S U(2)$. Here, we obtain a new larger game, $G^{Q_{\psi}}$, called the quantization of the game $G$ via the initial state $\psi$. The game $G^{Q_{\psi}}$ is completely specified by the quintuple

$$
G^{Q_{\psi}}=\left\{\psi ; S U(2), S U(2) ; P_{1}^{Q}, P_{2}^{Q}\right\}
$$

The function $P_{i}^{Q}: S U(2) \times S U(2) \rightarrow \Delta\left(\operatorname{Im} P_{i}\right)$ is referred to as the quantum payoff function for Player $i$.

Now, if we let the players use probabilistic mixtures of $S U(2)$ elements, we obtain a yet larger game $G^{m Q_{\psi}}$, the mixed quantization of $G$ with respect to the initial state $\psi$. This game is specified by

$$
G^{m Q_{\psi}}=\left\{\psi ; \Delta(S U(2)), \Delta(S U(2)) ; \in_{1}^{Q}, \in_{2}^{Q}\right\}
$$

The function $\in_{i}^{Q}: \Delta(S U(2)) \times \Delta(S U(2)) \rightarrow \Delta\left(\operatorname{Im} P_{i}\right)$ is referred to as the expected quantum payoff function for Player $i$. For more details on game extensions, the reader is referred to [2].

The actual computation of the payoffs that arise from a specific profile of players' choices of elements of $S U(2)$ or $\Delta(S U(2))$ will be discussed in details in the next two sections.

The fundamental goal in game theory, and hence in this paper, is to identify the Nash equilibria in the games 
$G, G^{\text {mix }}, G^{Q_{\psi}}$, and $G^{m Q_{\psi}}$.

Definition 1.1 Let $M$ be any of the games $G, G^{\text {mix }}, G^{Q_{\psi}}$, or $G^{m Q_{\psi}}$ and $S_{i}$ and $P_{i}$ be the associated strategy space and payoff function, respectively, for Player $i$. We say that a strategic profile $\left(u^{*}, v^{*}\right) \in S_{1} \times S_{2}$ is a Nash equilibrium or just an equilibrium in the game $M$ if

$$
P_{1}\left(u^{*}, v^{*}\right) \geq P_{1}\left(u, v^{*}\right) \forall u \in S_{1} \text { and } P_{2}\left(u^{*}, v^{*}\right) \geq P_{2}\left(u^{*}, v\right) \forall v \in S_{2}
$$

Other ways of expressing this concept include the observation that no player can increase his or her payoff by unilaterally deviating from his or her equilibrium strategy or that at the equilibrium a player's opponents are indifferent to that player's strategic choice. The existence of equilibria in a game in which the $S_{i}$ 's are all finite is guaranteed by Nash theorem [3].

\section{Quantum Payoff Function}

There are many quantization protocols in the literature, including some that utilize the initial state $\psi=\frac{|00\rangle+|11\rangle}{\sqrt{2}}$ [4]. In this paper, we consider two qubits with respect to the observational basis $\{|0\rangle,|1\rangle\}$ in the initial state given by Equation (1.9). The players operate on their respective qubits, the first via

$$
U_{I}=\left(\begin{array}{cc}
A & B \\
-\bar{B} & \bar{A}
\end{array}\right)
$$

and the second via

$$
U_{I I}=\left(\begin{array}{cc}
P & Q \\
-\bar{Q} & \bar{P}
\end{array}\right),
$$

respectively. Recall that the quantities $A, B, P$, and $Q$ are complex numbers subject to the normalization constraints $|A|^{2}+|B|^{2}=1$ and $|P|^{2}+|Q|^{2}=1$.

After the players act the initial state becomes with respect to the observational basis of the space of the joint states of the players' qubits

$$
\left(U_{I} \otimes U_{I I}\right)(\psi)=\frac{1}{\sqrt{2}}[(A P+i B Q)|00\rangle+(-A \bar{Q}+i B \bar{P})|01\rangle+(-\bar{B} P+i \bar{A} Q)|10\rangle+(\bar{B} \bar{Q}+i \bar{A} \bar{P})|11\rangle]
$$

We will refer to Equation (2.3) as the game state with respect to the observational basis. We consider next the actions No Flip and Flip represented by the $S U(2)$ matrices given by Equation (1.9). Note that $N(|0\rangle)=|0\rangle, N(|1\rangle)=|1\rangle, F(|0\rangle)=-\bar{\eta}|1\rangle \equiv|1\rangle$, and $F(|1\rangle)=\eta|1\rangle \equiv|0\rangle$. The last two equalities hold because the axioms of quantum mechanics stipulate that two states that differ by multiplication of a nonzero complex scalar, called a phase, are equal. So, in the joint observational basis $\{|00\rangle,|01\rangle,|10\rangle,|11\rangle\}$ we obtain that the game states corresponding to the action profiles are given by

$$
\begin{aligned}
& N N \equiv(N \otimes N)(\psi)=\frac{|00\rangle+i|11\rangle}{\sqrt{2}}, \quad N F \equiv(N \otimes F)(\psi)=\frac{-\bar{\eta}|01\rangle+i \eta|10\rangle}{\sqrt{2}}, \\
& F N \equiv(F \otimes N)(\psi)=\frac{i \eta|01\rangle-\bar{\eta}|10\rangle}{\sqrt{2}}, \quad F F \equiv(F \otimes F)(\psi)=\frac{i \eta^{2}|00\rangle+\bar{\eta}^{2}|11\rangle}{\sqrt{2}}
\end{aligned}
$$

Note that the foregoing vectors can be also expressed in matrix form as

$$
N N=\frac{1}{\sqrt{2}}\left(\begin{array}{l}
1 \\
0 \\
0 \\
i
\end{array}\right), N F=\frac{1}{\sqrt{2}}\left(\begin{array}{c}
0 \\
-\bar{\eta} \\
i \eta \\
0
\end{array}\right), F N=\frac{1}{\sqrt{2}}\left(\begin{array}{c}
0 \\
i \eta \\
-\bar{\eta} \\
0
\end{array}\right), F F=\frac{1}{\sqrt{2}}\left(\begin{array}{c}
i \eta^{2} \\
0 \\
0 \\
\bar{\eta}^{2}
\end{array}\right)
$$

For the purpose of the EWL protocols, these states are to correspond to a physical property observable to the referee. For this, the axioms of quantum mechanics require these states to form an orthogonal basis of the joint state space of the two qubits. For two elements $x$ and $y$ of an $n$-dimensional complex vector space $\mathbf{C}^{n}$, we use the 
inner product given by

$$
\langle x, y\rangle=\bar{x}^{\mathrm{T}} \cdot y
$$

Therefore, the set of vectors $\{N N, N F, F N, F F\}$ forms an orthogonal basis of the joint state space of the two qubits if and only if the vectors are pair wise orthogonal. The non-trivial orthogonality conditions are thus

$$
\langle N N, F F\rangle=\frac{1}{2}\left(i \eta^{2}-i \bar{\eta}^{2}\right)=0, \quad\langle N F, F N\rangle=\frac{1}{2}\left(-i \eta^{2}+i \bar{\eta}^{2}\right)=0
$$

Therefore, $\eta^{4}=1$. Thus setting $\eta=i$ insures the orthogonality of these states. For this specific value of $\eta=i$ the action basis vectors become

$$
N N=\frac{1}{\sqrt{2}}\left(\begin{array}{l}
1 \\
0 \\
0 \\
i
\end{array}\right), N F=\frac{1}{\sqrt{2}}\left(\begin{array}{c}
0 \\
i \\
-1 \\
0
\end{array}\right), F N=\frac{1}{\sqrt{2}}\left(\begin{array}{c}
0 \\
-1 \\
i \\
0
\end{array}\right), F F=\frac{1}{\sqrt{2}}\left(\begin{array}{c}
-i \\
0 \\
0 \\
-1
\end{array}\right)
$$

Therefore, after the players act, the game state becomes with respect to the action basis

$$
\begin{aligned}
\left(U_{I} \otimes U_{I I}\right)(\psi)= & \operatorname{Re}(A P+i B Q) N N+\operatorname{Re}(i A \bar{Q}+B \bar{P}) N F \\
& +\operatorname{Re}(A \bar{Q}-i B \bar{P}) F N+\operatorname{Re}(i A P-B Q) F F
\end{aligned}
$$

Hence, the referee observing the game state in the action basis sees each pure action state with probability given by

$$
\begin{aligned}
& \operatorname{prob}(N N)=[\operatorname{Re}(A P+i B Q)]^{2} \\
& \operatorname{prob}(N F)=[\operatorname{Re}(i A \bar{Q}+B \bar{P})]^{2} \\
& \operatorname{prob}(F N)=[\operatorname{Re}(A \bar{Q}-i B \bar{P})]^{2} \\
& \operatorname{prob}(F F)=[\operatorname{Re}(i A P-B Q)]^{2}
\end{aligned}
$$

This result leads to the following definition:

Definition 2.1 Let $G$ be the game described in Table 1. Then the associated quantization $G^{Q_{\psi}}$ with respect to the initial state $\psi$ is the two player game in which each player's strategy space is the set of special unitary matrices, $S U(2)$, and the quantum payoff functions for Player I and Player II are defined as follows:

$$
\begin{aligned}
& P_{1}^{Q}\left(U_{I}, U_{I I}\right)=[\operatorname{Re}(A P+i B Q)]^{2} x_{0}+[\operatorname{Re}(i A \bar{Q}+B \bar{P})]^{2} x_{3}+[\operatorname{Re}(A \bar{Q}-i B \bar{P})]^{2} x_{2}+[\operatorname{Re}(i A P-B Q)]^{2} x_{1} \\
& P_{2}^{Q}\left(U_{I}, U_{I I}\right)=[\operatorname{Re}(A P+i B Q)]^{2} y_{0}+[\operatorname{Re}(i A \bar{Q}+B \bar{P})]^{2} y_{3}+[\operatorname{Re}(A \bar{Q}-i B \bar{P})]^{2} y_{2}+[\operatorname{Re}(i A P-B Q)]^{2} y_{1}
\end{aligned}
$$

As Equations (2.10) - (2.12) indicate, the use of $S U(2)$ elements will prove impractical when one undertakes the important task of identifying potential quantum Nash equilibria of the game indicated in Table 1 . We will utilize the unit quaternions instead of the $S U(2)$ elements. The unit quaternions turn out to be more efficient and convenient in simplifying a great deal of equilibrium calculations.

First, we begin with a brief review of the real division algebra of the quaternions which can be also found in [5] and [6].

\section{Quaternions}

The quaternions, denoted by $\mathbf{H}$, are a 4-dimensional normed division algebra over the real numbers. They are spanned by the identity element 1 and three imaginary units $i, j$, and $k$. These fundamental units satisfy the so-called Hamilton's relation given by Equation (2.1). A general quaternion $q$ has form

$$
q=a+b i+c j+d k
$$


where $a, b, c$, and $d$ are real numbers. Addition and multiplication with quaternions are polynomial, subject to Hamilton's relation above. Multiplication with quaternions is not commutative but the distributive law holds. When $a=0$, that is, when $q=b i+c j+d k$, we call $q$ a pure quaternion. Each quaternion as above possesses a quaternionic conjugate $\bar{q}$ with $\bar{q}=a-b i-c j-d k$. The real-valued norm (or length) on the quaternions is defined by the formula

$$
N(q)=\sqrt{q \bar{q}}=\sqrt{a^{2}+b^{2}+c^{2}+d^{2}}
$$

One can easily verify that the norm of a product of quaternions is the product of their norms, that is,

$$
N(p q)=N(p) N(q)
$$

Each non-zero quaternion possesses a non-zero inverse

$$
q^{-1}=\frac{\bar{q}}{[N(q)]^{2}}
$$

This establishes the set of non-zero quaternions as division algebra. Moreover, the set of unit quaternions

$$
\mathrm{H}_{1}=\{q \mid N(q)=1\}
$$

forms a subgroup of $\mathbf{H}-\{\mathbf{0}\}$ under quaternionic multiplication and can be thought as the unit 3-sphere $\mathbf{S}^{3}$ living in $\mathbf{R}^{4}$. In light of the discussion above, one can see that the set of non-zero quaternions forms a skew-field.

We can also express a general quaternion in the form $p=\alpha+\beta j$, where $\alpha$ and $\beta$ are complex numbers. In this case, if $q=\delta+\gamma j$, then quaternionic multiplication is given by the map

$$
((\alpha, \beta),(\delta, \gamma)) \mapsto(\alpha \delta-\beta \bar{\gamma}, \alpha \gamma+\beta \bar{\delta})
$$

There are other identifications of $S^{3}$ that are of interest to us beyond that of the unit quaternions, in particular the identification of $\mathbf{S}^{3}$ with the special unitary group of two-by-two complex matrices, $S U(2)$. That is, those matrices with orthonormal columns and determinant 1 . This group isomorphism is given by the map

$$
\alpha+\beta j \mapsto\left(\begin{array}{cc}
\alpha & \beta \\
-\bar{\beta} & \bar{\alpha}
\end{array}\right),
$$

where the complex numbers $\alpha$ and $\beta$ are subject to the normalization condition $|\alpha|^{2}+|\beta|^{2}=1$.

For more details on real division algebras in general, and on quaternions in particular, the reader is referred to [7] [8].

\section{Unit Quaternions as Strategies}

There are many isomorphisms between the group $S U(2)$ of special unitary matrices and the group $\mathbf{S}^{3}$ of unit quaternions. We consider the following identifications.

Proposition 3.1.1 $S U(2)$ and $\mathbf{S}^{3}$ are isomorphic as groups via the identifications

$$
\left(\begin{array}{cc}
A & B \\
-\bar{B} & \bar{A}
\end{array}\right) \leftrightarrow p=A-i \mathrm{e}^{i \frac{\pi}{4}} B j
$$

and

$$
\left(\begin{array}{cc}
P & Q \\
-\bar{Q} & \bar{P}
\end{array}\right) \leftrightarrow q=P+i \mathrm{e}^{-i \frac{\pi}{4}} \bar{Q} j,
$$

where $p$ and $q \in \mathbf{S}^{3}$ and $A, B, P, Q$ are complex numbers such that $|A|^{2}+|B|^{2}=1$ and $|P|^{2}+|Q|^{2}=1$.

Proof: We prove that Equation (3.8) defines a group isomorphism. The proof that Equation (3.9) is a group isomorphism is similar but omitted. Define the mapping $\varphi: S U(2) \rightarrow \mathbf{S}^{3}$ by $\varphi\left(\left(\begin{array}{cc}x & y \\ -\bar{y} & \bar{x}\end{array}\right)\right)=x-i \mathrm{e}^{i \frac{\pi}{4}} y j$. First, 
we show that $p=x-i e^{i \frac{\pi}{4}} y j$ is a unit quaternion. For this, set $x=x_{0}+x_{1} i$ and $y=y_{0}+y_{1} i$. Then

$$
\begin{aligned}
p & =\left(x_{0}+x_{1} i\right)-i\left(\frac{\sqrt{2}}{2}+i \frac{\sqrt{2}}{2}\right)\left(y_{0}+y_{1} i\right) j \\
& =x_{0}+x_{1} i+\frac{\sqrt{2}}{2}\left(y_{0}+y_{1}\right) j-\frac{\sqrt{2}}{2}\left(y_{0}-y_{1}\right) k
\end{aligned}
$$

and has norm

$$
\begin{aligned}
N(p) & =\sqrt{x_{0}^{2}+x_{1}^{2}+\frac{1}{2}\left[y_{0}+y_{1}\right]^{2}+\frac{1}{2}\left[y_{0}-y_{1}\right]^{2}} \\
& =\sqrt{x_{0}^{2}+x_{1}^{2}+y_{0}^{2}+y_{1}^{2}}=1
\end{aligned}
$$

since the $S U(2)$ element $\left(\begin{array}{cc}x & y \\ -\bar{y} & \bar{x}\end{array}\right)$ has the property that $|x|^{2}+|y|^{2}=x_{0}^{2}+x_{1}^{2}+y_{0}^{2}+y_{1}^{2}=1$. This shows that the image of an $S U(2)$ element under the mapping $\varphi$ is a unit quaternion.

Next, we show that the mapping $\varphi$ is a group homomorphism. For this, let $M=\left(\begin{array}{cc}x & y \\ -\bar{y} & \bar{x}\end{array}\right)$ and $N=\left(\begin{array}{cc}u & v \\ -\bar{v} & \bar{u}\end{array}\right)$ be arbitrary elements of $S U(2)$. Then, on one hand

$$
\varphi(M N)=\varphi\left(\left[\begin{array}{cc}
x u-y \bar{v} & x v+y \bar{u} \\
-\bar{y} u-\overline{x u} & -\bar{y} v+\overline{x u}
\end{array}\right]\right)=(x u-y \bar{v})-i \mathrm{e}^{i \frac{\pi}{4}}(x v+y \bar{u}) j
$$

and on the other hand

$$
\varphi(M) \varphi(N)=\left(x-i \mathrm{e}^{i \frac{\pi}{4}} y j\right)\left(u-i \mathrm{e}^{i \frac{\pi}{4}} v j\right)=(x u-y \bar{u})-i \mathrm{e}^{i \frac{\pi}{4}}[x v+y \bar{u}] j
$$

Hence, $\varphi(M N)=\varphi(M) \varphi(N)$ for all $M, N \in S U(2)$. It remains to show that $\varphi$ is one-to-one and onto. Note that $\varphi(M)=1$ yields $x-i \mathrm{e}^{i \frac{\pi}{4}} y j=1$ or $x_{0}+x_{1} i+\frac{\sqrt{2}}{2}\left[y_{0}+y_{1}\right] j-\frac{\sqrt{2}}{2}\left[y_{0}-y_{1}\right] k=1$.

This can happen if and only if $x_{0}=1, x_{1}=0, y_{0}+y_{1}=0$ and $y_{0}-y_{1}=0$, that is, $x_{0}=1$ and $x_{1}=y_{0}=y_{1}=0$. Therefore, $\operatorname{Ker} \varphi=\left\{\left(\begin{array}{ll}1 & 0 \\ 0 & 1\end{array}\right)\right\}$ which proves that $\varphi$ is one-to-one.

Finally, note that each unit quaternion $p=a_{0}+a_{1} i+a_{2} j+a_{3} k$ has the $S U(2)$ element $\left(\begin{array}{cc}\alpha & \beta \\ -\bar{\beta} & \bar{\alpha}\end{array}\right)$, where $\alpha=a_{0}+a_{1} i$ and $\beta=\frac{1}{\sqrt{2}}\left[\left(a_{2}-a_{3}\right)+\left(a_{2}+a_{3}\right) i\right]$, as a pre-image under the map $\varphi$. Therefore, $\varphi$ is onto. This completes the proof that $\varphi: S U(2) \rightarrow \mathbf{S}^{3}$ is a group isomorphism.

Now we take $p$ and $q$ as given in Equations (3.8) and (3.9) and compute the product

$$
\begin{aligned}
p q & =\left(A-i \mathrm{e}^{i \frac{\pi}{4}} B j\right)\left(P+i \mathrm{e}^{-i \frac{\pi}{4}} \bar{Q} j\right) \\
& =A P+i \mathrm{e}^{-i \frac{\pi}{4}} A \bar{Q} j-i \mathrm{e}^{i \frac{\pi}{4}} B j \cdot i \mathrm{e}^{-i \frac{\pi}{4}} \bar{Q} j-i \mathrm{e}^{i \frac{\pi}{4}} B j P \\
& =A P+i B Q+\left(\mathrm{e}^{i \frac{\pi}{4}} A \bar{Q}+\mathrm{e}^{-i \frac{\pi}{4}} B \bar{P}\right) j
\end{aligned}
$$

This product can be also expressed as 


$$
\begin{aligned}
p q= & (\operatorname{Re}[A P+i B Q]) \cdot 1+(\operatorname{Im}[A P+i B Q]) i \\
& +(\operatorname{Re}[i A \bar{Q}+B \bar{P}])\left(\mathrm{e}^{-i \frac{\pi}{4}} j\right)+(\operatorname{Im}[i A \bar{Q}+B \bar{P}])\left(\mathrm{e}^{-i \frac{\pi}{4}} k\right) \\
= & \pi_{0}(p q) \cdot 1+\pi_{1}(p q) \cdot i+\pi_{2}(p q) \cdot\left(\mathrm{e}^{-i \frac{\pi}{4}} j\right)+\pi_{3}(p q) \cdot\left(\mathrm{e}^{i \frac{\pi}{4}} k\right)
\end{aligned}
$$

where $\pi_{0}(p q)=(\operatorname{Re}[A P+i B Q]), \quad \pi_{1}(p q)=(\operatorname{Im}[A P+i B Q])$, and $\pi_{3}(p q)=(\operatorname{Im}[i A \bar{Q}+B \bar{P}])$.

To further simplify the product $p q$, we utilize the following straightforward identities

(1) $\operatorname{Im}(A P+i B Q)=-\operatorname{Re}(i A P-B Q)$

(2) $\operatorname{Im}(i A \bar{Q}+B \bar{P})=-\operatorname{Re}(-A \bar{Q}+i B \bar{P})=\operatorname{Re}(A \bar{Q}-i B \bar{P})$

Therefore the expression for $p q$ simplifies to

$$
\begin{aligned}
p q= & (\operatorname{Re}[A P+i B Q]) \cdot 1+(-\operatorname{Re}[i A P-B Q]) i \\
& +(\operatorname{Re}[i A \bar{Q}+B \bar{P}])\left(\mathrm{e}^{-i \frac{\pi}{4}} j\right)+(\operatorname{Re}[A \bar{Q}-i B \bar{P}])\left(\mathrm{e}^{-i \frac{\pi}{4}} k\right)
\end{aligned}
$$

Comparing Equations (2.10) and (3.16), we conclude that

$$
\begin{aligned}
& \operatorname{prob}(N N)=[\operatorname{Re}(A P+i B Q)]^{2}=\left[\pi_{0}(p q)\right]^{2} \\
& \operatorname{prob}(N F)=[\operatorname{Re}(i A \bar{Q}+B \bar{P})]^{2}=\left[\pi_{2}(p q)\right]^{2} \\
& \operatorname{prob}(F N)=[\operatorname{Re}(A \bar{Q}-i B \bar{P})]^{2}=\left[\pi_{3}(p q)\right]^{2} \\
& \operatorname{prob}(F F)=[\operatorname{Re}(i A P-B Q)]^{2}=\left[\pi_{1}(p q)\right]^{2}
\end{aligned}
$$

This result motivates the following definition.

Definition 3.1.2 Let G be the game depicted in Table 1. Then the corresponding pure quantum game with respect to the initial state $\psi, G^{Q_{\psi}}$, is the two player game in which each player's strategy space is the set of unit quaternions with basis $B_{1}=\left\{1, i, \mathrm{e}^{-i \frac{\pi}{4}} j, \mathrm{e}^{-i \frac{\pi}{4}} k\right\}$ and Player I's and Player II's payoff functions are given by

$$
\begin{aligned}
& P_{1}^{Q}(p, q)=\sum_{t=0}^{3}\left[\pi_{t}(p q)\right]^{2} x_{t} \\
& P_{2}^{Q}(p, q)=\sum_{t=0}^{3}\left[\pi_{t}(p q)\right]^{2} y_{t}
\end{aligned}
$$

We observe that the basis $B_{1}=\left\{1, i, \mathrm{e}^{-i \frac{\pi}{4}} j, \mathrm{e}^{-i \frac{\pi}{4}} k\right\}$ is an orthonormal basis of $\mathbf{H}$. In practice, the players would love to use unit quaternions that are linear combinations of the elements of the canonical basis of $\mathbf{H}$, $B_{0}=\{1, i, j, k\}$. An easy exercise is to observe that the basis change matrix from basis $B_{1}$ to basis $B_{0}$ is

$$
M=\left(\begin{array}{cccc}
1 & 0 & 0 & 0 \\
0 & 1 & 0 & 0 \\
0 & 0 & \frac{\sqrt{2}}{2} & -\frac{\sqrt{2}}{2} \\
0 & 0 & \frac{\sqrt{2}}{2} & \frac{\sqrt{2}}{2}
\end{array}\right)
$$

This matrix is unitary, therefore the basis change matrix from $B_{0}$ to $B_{1}$ is 


$$
M^{-1}=\bar{M}^{\mathrm{T}}=\left(\begin{array}{cccc}
1 & 0 & 0 & 0 \\
0 & 1 & 0 & 0 \\
0 & 0 & \frac{\sqrt{2}}{2} & \frac{\sqrt{2}}{2} \\
0 & 0 & -\frac{\sqrt{2}}{2} & \frac{\sqrt{2}}{2}
\end{array}\right)
$$

If the players employ unit quaternions based on $1, i, j$, and $k$ then the payoffs to Player I and Player II are

$$
\begin{gathered}
P_{1}^{Q}(p, q)=\sum_{t=0}^{3}\left[\pi_{t}\left(M^{-1}(p q)\right)\right]^{2} x_{t} \\
P_{2}^{Q}(p, q)=\sum_{t=0}^{3}\left[\pi_{t}\left(M^{-1}(p q)\right)\right]^{2} y_{t}
\end{gathered}
$$

respectively, where $x_{t}$ and $y_{t}$ are taken from Table 1 . These results are summarized in the following definition.

Definition 3.1.3 Let $G$ be the game depicted in Table 1. Then the corresponding pure quantum game with respect to the initial state $\psi, G^{Q_{\psi}}$, is the two player game in which each player's strategy space is the set of unit quaternions with basis $B_{0}=\{1, i, j, k\}$ and Player I's and Player II's payoff functions are given by Equations (3.21) and (3.22), respectively.

We conclude this section with the observation that the map $P_{1}^{Q}: \mathbf{H}_{1} \times \mathbf{H}_{2} \rightarrow \Delta\left(\operatorname{Im} P_{i}\right)$ is onto as opposed to the map $\epsilon_{i}:[0,1] \times[0,1] \rightarrow \Delta\left(\operatorname{Im} P_{i}\right)$ of Section 1 . Indeed, for any probability distribution over the outcomes of the game $G$ described in Table 1, $p_{0}\left(x_{0}, y_{0}\right)+p_{1}\left(x_{1}, y_{1}\right)+p_{2}\left(x_{2}, y_{2}\right)+p_{3}\left(x_{3}, y_{3}\right)$, there are unit quaternions $p$ and $q$ such that $\pi_{0}^{2}(p q)=p_{0}, \pi_{1}^{2}(p q)=p_{1}, \pi_{2}^{2}(p q)=p_{2}$, and $\pi_{3}^{2}(p q)=p_{3}$.

It is sufficient to choose $p=1$ and $q=\sqrt{p_{0}}+\sqrt{p_{1}} i+\frac{\sqrt{2}}{2}\left(\sqrt{p_{2}}+\sqrt{p_{3}}\right) j+\frac{\sqrt{2}}{2}\left(\sqrt{p_{2}}-\sqrt{p_{3}}\right) k$.

\section{Mixed Quantum Strategies}

A mixed quantum strategy is a probability distribution over the set of unit quaternions.

While the consideration of the entire space of mixed quantum strategies remains a goal of a future work, the use of mixed quantum strategies supported on the canonical basis elements $1, i, j$ and $k$, that is, elements of the form

$$
a_{0}+a_{1} i+a_{2} j+a_{3} k, \quad 0 \leq a_{t} \leq 1 \text { and } \sum_{t=0}^{3} a_{t}=1,
$$

has already established some interesting results.

Definition 4.1 Let G be the game depicted in Table 1. Then the corresponding mixed quantum game with respect to the initial state $\psi, G^{m Q_{\psi}}$, is the two player game in which each player's strategy space is the set of probability distributions over the unit quaternions and Player $i$ 's expected payoff function is

$\epsilon_{i}^{Q}: \Delta\left(S^{3}\right) \times \Delta\left(S^{3}\right) \rightarrow \Delta\left(\operatorname{Im} P_{i}\right)$.

As we did in the game $G^{Q}$, we will derive equations for the players' expected payoff functions in the game $G^{m Q_{\psi}}$. For this, suppose Player I uses the mixed quantum strategy

$$
\mu=a_{0}+a_{1} i+a_{2} j+a_{3} k
$$

and Player II uses the mixed quantum strategy

$$
v=b_{0}+b_{1} i+b_{2} j+b_{3} k
$$

Then the expected payoff to Player I is given by

$$
\begin{aligned}
\epsilon_{1}^{Q}(\mu, v)= & a_{0}\left[b_{0} x_{0}+b_{1} x_{1}+b_{2}\left(\frac{x_{2}+x_{3}}{2}\right)+b_{3}\left(\frac{x_{2}+x_{3}}{2}\right)\right]+a_{1}\left[b_{0} x_{1}+b_{1} x_{0}+b_{2}\left(\frac{x_{2}+x_{3}}{2}\right)+b_{3}\left(\frac{x_{2}+x_{3}}{2}\right)\right] \\
& +a_{2}\left[b_{0}\left(\frac{x_{2}+x_{3}}{2}\right)+b_{1}\left(\frac{x_{2}+x_{3}}{2}\right)+b_{2} x_{0}+b_{3} x_{1}\right]+a_{3}\left[b_{0}\left(\frac{x_{2}+x_{3}}{2}\right)+b_{1}\left(\frac{x_{2}+x_{3}}{2}\right)+b_{2} x_{1}+b_{3} x_{0}\right]
\end{aligned}
$$


or, in matrix form

$$
\epsilon_{1}^{Q}(\mu, v)=\left(\begin{array}{llll}
a_{0} & a_{1} & a_{2} & a_{3}
\end{array}\right)\left(\begin{array}{cccc}
x_{0} & x_{1} & \frac{x_{2}+x_{3}}{2} & \frac{x_{2}+x_{3}}{2} \\
x_{1} & x_{0} & \frac{x_{2}+x_{3}}{2} & \frac{x_{2}+x_{3}}{2} \\
\frac{x_{2}+x_{3}}{2} & \frac{x_{2}+x_{3}}{2} & x_{0} & x_{1} \\
\frac{x_{2}+x_{3}}{2} & \frac{x_{2}+x_{3}}{2} & x_{1} & x_{0}
\end{array}\right)\left(\begin{array}{l}
b_{0} \\
b_{1} \\
b_{2} \\
b_{3}
\end{array}\right)
$$

Player II's expected payoff is derived in a similar manner, except that the letter $x$ is replaced with the letter $y$.

We note here the similarities between the quantum expected payoff function in $G^{m_{Q_{\mu}}}$ (Equation (4.5)) and the classical expected payoff function in $G^{\text {mix }}$ (Equation (1.6)).

Note that we can also express Equation (4.4) in the form

$$
\epsilon_{1}^{Q}(\mu, v)=a_{0} A_{0}+a_{1} A_{1}+a_{2} A_{2}+a_{3} A_{3},
$$

and similarly

$$
\epsilon_{2}^{Q}(\mu, v)=b_{0} B_{0}+b_{1} B_{1}+b_{2} B_{2}+b_{3} B_{3} .
$$

Equation (4.7) is the expected quantum payoff function for Player II. We will refer to the coefficients $a_{t}$ and $b_{t}$ as the frequencies and the numbers $A_{t}$ and $B_{t}$ as the returns.

\section{Application}

As an application of the theory discussed in the previous sections, we consider a variant of the game of Chicken with bimatrix given by Table 2 .

In the Game of Chicken, the expected payoff to Player I is given by

$$
\epsilon_{1}(p, q)=p[2 q]+(1-p)[4 q-1]
$$

with corresponding best reply correspondence $R_{1}:[0,1] \rightarrow[0,1]$ given by

$$
R_{1}(q)= \begin{cases}1, & \text { if } 0 \leq q<1 / 2 \\ {[0,1],} & \text { if } q=1 / 2 \\ 0, & \text { if } 1 / 2<q \leq 1\end{cases}
$$

Similarly, Player II's expected payoff function is given by

$$
\epsilon_{2}(p, q)=q[2 p]+(1-q)[4 p-1]
$$

with corresponding best reply correspondence $R_{2}:[0,1] \rightarrow[0,1]$ given by

$$
R_{2}(p)= \begin{cases}1, & \text { if } 0 \leq p<1 / 2 \\ {[0,1],} & \text { if } p=1 / 2 \\ 0, & \text { if } 1 / 2<p \leq 1\end{cases}
$$

The reaction curves that represent these correspondences are shown in Figure 1, with the dashed lines for Player I's reaction curve and the solid lines for Player II's reaction curve.

It is well known that in any variant of the game of chicken there are always three classical equilibria, two in pure strategies and one in mixed strategies. Nash equilibria occur where the reaction curves intersect. From Figure 1, one can see that the classical pure equilibria are $(p=0, q=1)$ and $(p=1, q=0)$ or, equivalently, the pure strategy pairs $\left(s_{2}, t_{1}\right)$ and $\left(s_{1}, t_{2}\right)$ with corresponding payoffs $(3,0)$ and $(0,3)$ to the players, respectively. The equilibrium in mixed strategies is the pair $\left(\frac{1}{2}, \frac{1}{2}\right)$. This equilibrium pays out each player an expected payoff of 1 . 


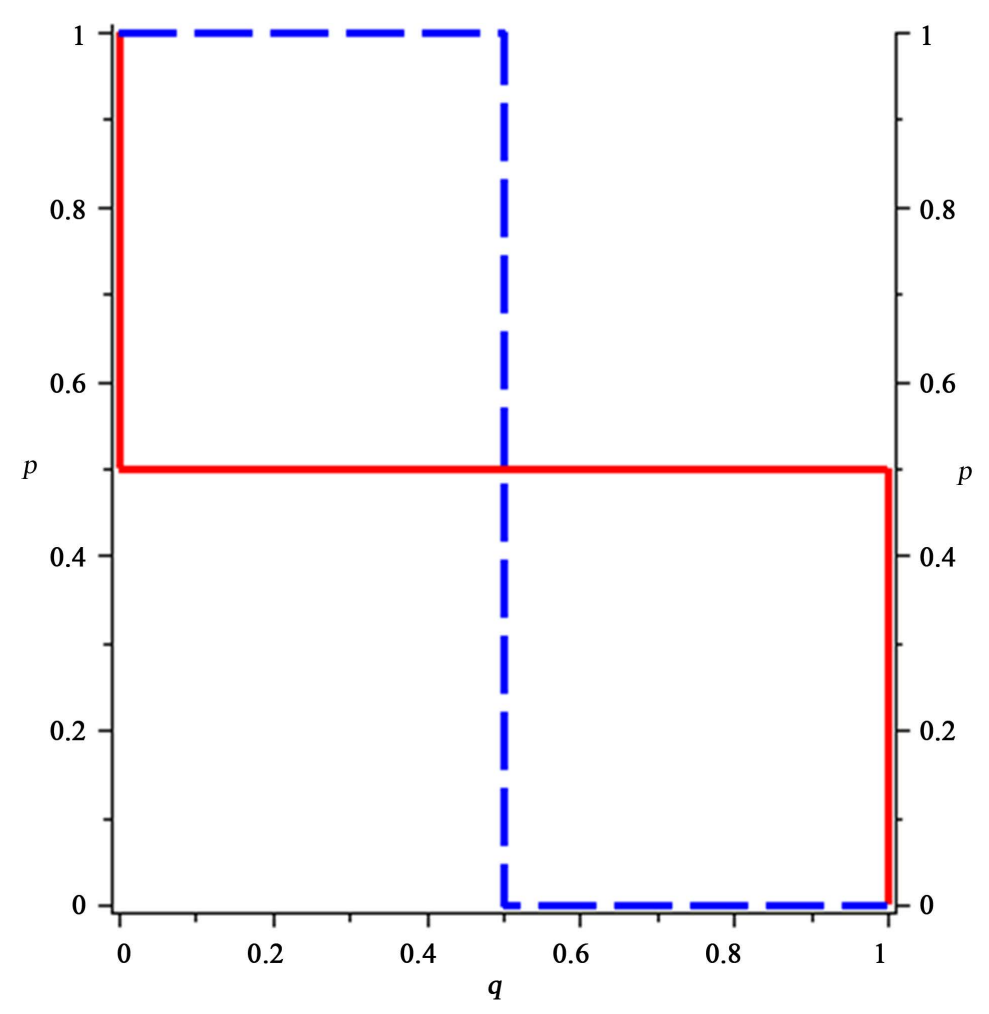

Figure 1. Reaction curves in the game of chicken.

However, in contrast to the classical situation, we find, using best response analysis, that the quantized version possesses many Nash equilibria.

\subsection{Equilibria of Type (Pure, Pure)}

There are four equilibria where each player employs a pure quantum strategy represented by a canonical quaternionic basis element.

Proposition 5.1 The Game of Chicken described in Table 2 admits the following Nash equilibria in pure quantum strategies:

Moreover, these equilibria pay out each player an expected payoff of 2 .

Proof: If Players I and II employ the mixed quantum strategies $\mu=a_{0}+a_{1} i+a_{2} j+a_{3} k$ and $v=b_{0}+b_{1} i+b_{2} j+b_{3} k$, respectively, then, by Equation (4.4), the expected payoffs to the players are

$$
\begin{aligned}
\epsilon_{1}^{Q}(\mu, v)= & a_{0}\left[2 b_{0}-b_{1}+\frac{3}{2} b_{2}+\frac{3}{2} b_{3}\right]+a_{1}\left[-b_{0}+2 b_{1}+\frac{3}{2} b_{2}+\frac{3}{2} b_{3}\right] \\
& +a_{2}\left[\frac{3}{2} b_{0}+\frac{3}{2} b_{1}+2 b_{2}-b_{3}\right]+a_{3}\left[\frac{3}{2} b_{0}+\frac{3}{2} b_{1}-b_{2}+2 b_{3}\right] \\
= & a_{0} A_{0}+a_{1} A_{1}+a_{2} A_{2}+a_{3} A_{3}
\end{aligned}
$$

and 
Table 2. Game of chicken.

\begin{tabular}{cccc}
\hline & \multicolumn{3}{c}{ Player II } \\
\cline { 2 - 4 } Player I & $t_{1}$ & $t_{2}$ \\
\cline { 2 - 4 } & $s_{1}$ & $(2,2)$ & $(0,3)$ \\
& $s_{2}$ & $(3,0)$ & $(-1,-1)$ \\
\hline
\end{tabular}

$$
\begin{aligned}
\in_{2}^{Q}(\mu, v)= & b_{0}\left[2 a_{0}-a_{1}+\frac{3}{2} a_{2}+\frac{3}{2} a_{3}\right]+b_{1}\left[-a_{0}+2 a_{1}+\frac{3}{2} a_{2}+\frac{3}{2} a_{3}\right] \\
& +b_{2}\left[\frac{3}{2} a_{0}+\frac{3}{2} a_{1}+2 a_{2}-a_{3}\right]+b_{3}\left[\frac{3}{2} a_{0}+\frac{3}{2} a_{1}-a_{2}+2 a_{3}\right] \\
= & b_{0} B_{0}+b_{1} B_{1}+b_{2} B_{2}+b_{3} B_{3}
\end{aligned}
$$

Suppose that $A_{0}$ is the largest return. Then Player I will choose a $\mu$ that concentrates all the frequencies on $A_{0}$, that is $a_{0}=1, a_{1}=a_{2}=a_{3}=0$, or $\mu=1$. Therefore, Player II's expected payoff becomes

$$
\epsilon_{2}^{Q}(\mu, v)=b_{0}(2)+b_{1}(-1)+b_{2}\left(\frac{3}{2}\right)+b_{3}\left(\frac{3}{2}\right)
$$

It is clear that Player II's expected payoff is maximal when he or she responds with a $v$ that concentrates all the frequencies on the largest return 2, that is, $b_{0}=1, b_{1}=b_{2}=b_{3}=0$ and therefore $v=1$ is the best reply to Player I's choice of $\mu=1$. Now suppose that Player II selects the unit quaternion $v=1$ as a strategy. Then Player I's expected payoff becomes

$$
\epsilon_{1}^{Q}(\mu, v)=a_{0}(2)+a_{1}(-1)+a_{2}\left(\frac{3}{2}\right)+a_{3}\left(\frac{3}{2}\right) .
$$

Then Player I's best response will be to select $\mu=1$. Therefore, the pure quantum strategic pair $(\mu, v)=(1,1)$ is a Nash equilibrium with the expected payoff of $\epsilon_{1}^{Q}(1,1)=\epsilon_{2}^{Q}(1,1)=2$ to the players. In a similar manner, when $A_{t}, t=1,2,3$ is maximal, one verifies that the pure quantum strategic pairs $(i, i),(j, j)$, and $(k, k)$ are Nash equilibria with an expected payoff of 2 to each player.

Equilibria in mixed quantum strategies occur when two or more returns are equal and maximal.

\subsection{Equilibria of Type (Mix of 2, Mix of 2)}

We begin with the situation where two returns are equal and maximal.

Proposition 5.2 The Game of Chicken admits the following Nash equilibria in mixed quantum strategies:

$$
\begin{aligned}
& \left(a_{0}+a_{1} i, b_{2} j+b_{3} k\right) \text { with } a_{0}, a_{1}, b_{2}, b_{3} \in(1 / 6,5 / 6) \\
& \left(\frac{1}{2}+\frac{1}{2} j, \frac{1}{2}+\frac{1}{2} j\right) \\
& \left(\frac{1}{2}+\frac{1}{2} k, \frac{1}{2}+\frac{1}{2} k\right) \\
& \left(\frac{1}{2} i+\frac{1}{2} j, \frac{1}{2} i+\frac{1}{2} j\right) \\
& \left(\frac{1}{2} i+\frac{1}{2} k, \frac{1}{2} i+\frac{1}{2} k\right) \\
& \left(a_{2} j+a_{3} k, b_{0}+b_{1} i\right) \text { with } a_{2}, a_{3}, b_{0}, b_{1} \in(1 / 6,5 / 6)
\end{aligned}
$$

Moreover, all these equilibria pay out each player an expected payoff of 7/4, except the equilibria given by (5.13) and (5.18) which pay out each player an expected payoff of 1.5. 
Proof: We begin by proving that the strategic pair given in Equation (5.13) is a Nash equilibrium. For this, suppose that the returns $A_{0}$ and $A_{1}$ are equal and maximal. Then, Player I will select $\mu=a_{0}+a_{1} i$, that is, $a_{0}+a_{1}=1$ and $a_{2}=a_{3}=0$. Therefore, Player II's expected payoff becomes

$$
\epsilon_{2}^{Q}(\mu, v)=b_{0}\left(3 a_{0}-1\right)+b_{1}\left(-a_{0}+2 a_{1}\right)+b_{2}\left(\frac{3}{2}\right)+b_{3}\left(\frac{3}{2}\right)
$$

To determine the largest return, we graph $B_{0}=3 a_{0}-1, B_{1}=2-3 a_{0}$, and $B_{2}=B_{3}=3 / 2$ against the frequency $a_{0}$, see Figure 2 .

From the graphs, we see that Player II will respond with $v=1, i, j$, or $k$ when $a_{0} \in\left[0, \frac{1}{6}\right] \cup\left[\frac{5}{6}, 1\right]$. However, from Proposition 5.1, we know that a best reply to $v=1, i, j$, or $k$ is $\mu=1, i, j$, or $k$, respectively. So we disregard the cases $0 \leq a_{0} \leq \frac{1}{6}$ and $\frac{5}{6} \leq a_{0} \leq 1$. When $\frac{1}{6}<a_{0}<\frac{5}{6}$, the largest returns are $B_{2}=B_{3}=\frac{3}{2}$. Hence, Player II will respond with $v=b_{2} j+b_{3} k$.

Now suppose that Player 2 selects the mixed quantum strategy $v=b_{2} j+b_{3} k$ together with the condition that $\frac{1}{6}<a_{0}<\frac{5}{6}$. Then, Player I's expected payoff becomes

$$
\epsilon_{1}^{Q}(\mu, v)=a_{0}\left(\frac{3}{2}\right)+a_{1}\left(\frac{3}{2}\right)+a_{2}\left(3 b_{2}-1\right)+a_{3}\left(2-3 b_{2}\right)
$$

Figure 3 shows the graphs of the returns $A_{0}=A_{1}=\frac{3}{2}, A_{2}=3 b_{2}-1$, and $A_{3}=2-3 b_{2}$ against the frequency $b_{2}$.

From the graphs, we see that Player I will respond with $\mu=1, i, j$, or $k$ when $b_{2} \in\left[0, \frac{1}{6}\right] \cup\left[\frac{5}{6}, 1\right]$. This contradicts the condition $\frac{1}{6}<a_{0}<\frac{5}{6}$. So we disregard the cases $0 \leq b_{2}<\frac{1}{6}$ and $\frac{5}{6}<b_{2} \leq 1$. When $\frac{1}{6}<b_{2}<\frac{5}{6}$, the largest returns are $A_{0}=A_{1}=\frac{3}{2}$. Hence, Player I will respond with $\mu=a_{0}+a_{1} i$.

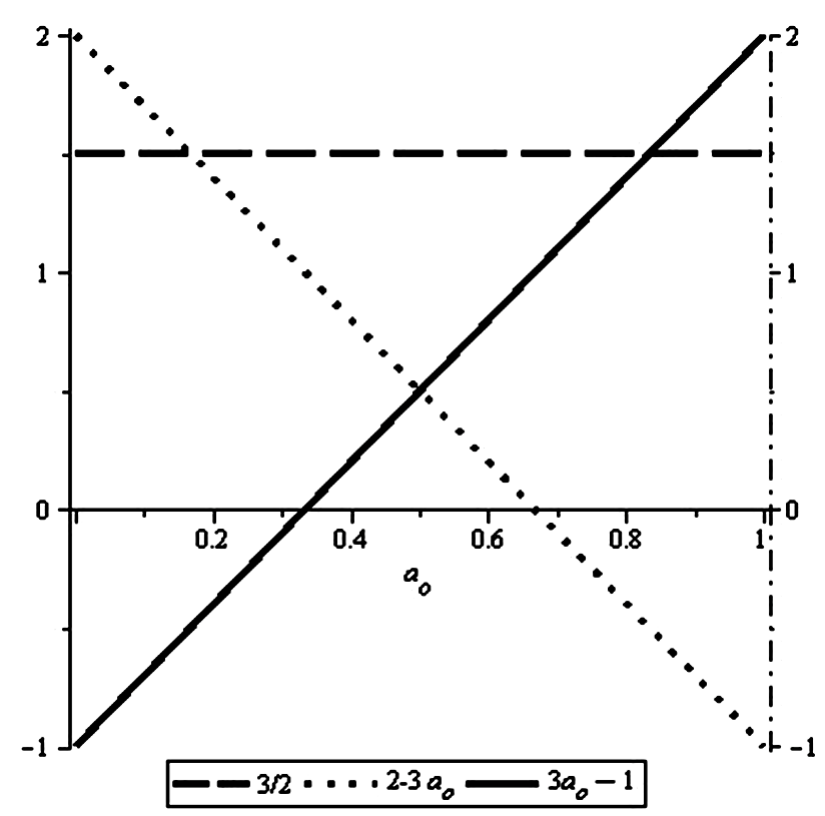

Figure 2. Graphs of the returns $B_{0}, B_{1}, B_{2}$ and $B_{3}$. 


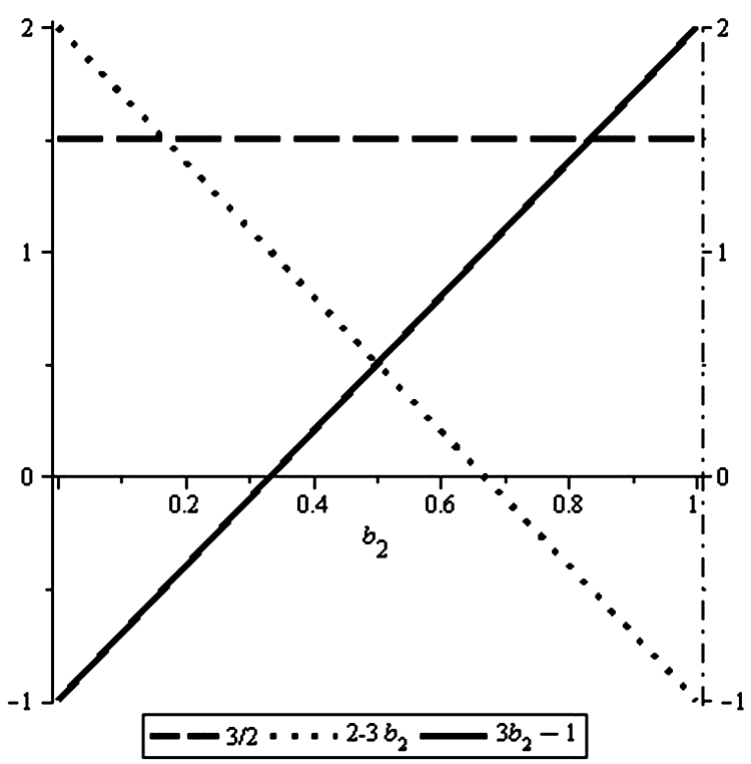

Figure 3. Graphs of the returns $A_{0}, A_{1}, A_{2}$ and $A_{3}$.

Therefore, we have a family of equilibria given by the strategic pair $(\mu, v)=\left(a_{0}+a_{1} i, b_{2} j+b_{3} k\right)$ with the constraints that $\frac{1}{6}<a_{0}<\frac{5}{6}$ and $\frac{1}{6}<b_{2}<\frac{5}{6}$. This family of equilibria pays out each player an expected payoff of

$$
\epsilon_{1}^{Q}\left(a_{0}+a_{1} i, b_{2} j+b_{3} k\right)=\epsilon_{2}^{Q}\left(a_{0}+a_{1} i, b_{2} j+b_{3} k\right)=\frac{3}{2} .
$$

In a similar manner, One verifies that the strategic pairs given in (5.14) - (5.18) are Nash equilibria.

\subsection{Equilibria of Type (Mix of 3, Mix of 3)}

When three returns are maximal and equal, we obtain four cases to study. For this particular game, it turns out that there are no equilibria of type mix of 3 against mix of 3.

\subsection{All the Returns Are Equal}

Proposition 5.3 The mixed quantum strategic pair

$$
(\mu, v)=\left(\frac{1}{4}+\frac{1}{4} i+\frac{1}{4} j+\frac{1}{4} k, \frac{1}{4}+\frac{1}{4} i+\frac{1}{4} j+\frac{1}{4} k\right)
$$

is a Nash equilibrium with expected payoffs to the players given by $\epsilon_{1}^{Q}(\mu, v)=\epsilon_{2}^{Q}(\mu, v)=1$.

Proof: When all the returns are equal, Player I is indifferent between all his/her mixed strategies and, therefore, can select any probability distribution over the returns $A_{0}, A_{1}, A_{2}$, and $A_{3}$. The equations $A_{0}=A_{1}=A_{2}=A_{3}$ yield the system of linear equations

$$
\left\{\begin{array}{l}
2 b_{0}-b_{1}+\frac{3}{2} b_{2}+\frac{3}{2} b_{3}=\lambda \\
-b_{0}+2 b_{1}+\frac{3}{2} b_{2}+\frac{3}{2} b_{3}=\lambda \\
\frac{3}{2} b_{0}+\frac{3}{2} b_{1}+2 b_{2}-b_{3}=\lambda \\
\frac{3}{2} b_{0}+\frac{3}{2} b_{1}-b_{2}+2 b_{3}=\lambda
\end{array},\right.
$$


where $\lambda$ is a fixed real number. This system has the unique solution

$$
b_{0}=\frac{1}{4} \lambda, b_{1}=\frac{1}{4} \lambda, b_{2}=\frac{1}{4} \lambda, b_{3}=\frac{1}{4} \lambda \text {. }
$$

Since $b_{0}+b_{1}+b_{2}+b_{3}=1$, we obtain $b_{0}=b_{1}=b_{2}=b_{3}=\frac{1}{4}$.

Now suppose Player II uses the mixed quantum strategy $v=\frac{1}{4}+\frac{1}{4} i+\frac{1}{4} j+\frac{1}{4} k$. Then Player I's expected payoff becomes $\epsilon_{1}^{Q}(\mu, v)=a_{0}+a_{1}+a_{2}+a_{3}=1$. Hence Player I is indifferent between all his/her probability distributions. In particular, he/she can respond with $a_{0}=a_{1}=a_{2}=a_{3}=\frac{1}{4}$. Therefore, the mixed quantum strategic pair $(\mu, v)=\left(\frac{1}{4}+\frac{1}{4} i+\frac{1}{4} j+\frac{1}{4} k, \frac{1}{4}+\frac{1}{4} i+\frac{1}{4} j+\frac{1}{4} k\right)$ is a Nash equilibrium with expected payoffs to the players given by $\epsilon_{1}^{Q}(\mu, v)=\epsilon_{2}^{Q}(\mu, v)=1$.

\section{Conclusions}

It often happens that the players' reaction curves cross several times as is the case in the game of Chicken. The classical version of the game of Chicken has three equilibria. Which of these should be selected? Only the equilibrium in mixed strategies that pays out each player a payoff of 1 makes sense for rational players, as the selection of one of the remaining two classical equilibria will result in one of the players being worse off. We, therefore, observe that the quantum payoffs are all equal or superior to the classical payoff $(1,1)$ that is desired by both players.

Moreover, comparing our findings to known results, we observe that the strategic pair $(\mu, v)=(1 / 2+(1 / 2) j, 1 / 2+(1 / 2) j)$ is also an equilibrium in Landsburg's quantized game of Chicken with expected payoffs of $(2.5,1)$ to the players [4]. Recall that, in our game, the payoff to each player at this equilibrium is 7/4. Thus, Player II is better off playing Chicken when it is quantized via our initial state.

The groundwork for a parallel extension of this work to three player, two strategy games can be found in [9] and [10]. A future direction of this work is the complete classification of potential Nash equilibria in three player, two strategy quantum games.

\section{Acknowledgements}

The authors would like to thank the referees for some valuable comments and helpful suggestions.

\section{References}

[1] Eisert, J., Wilkens, M. and Lewenstein, M. (1999) Quantum Games and Quantum Strategies. Physical Review Letters, 83, 3077-3080. http://dx.doi.org/10.1103/PhysRevLett.83.3077

[2] Bleiler, S.A. (2008) A Formalism for Quantum Games and an Application. Proceedings to the 9th International Pure Math Conference, Islamabad.

[3] Nash, J. (1950) Equilibrium Points in N-Person Games. Proceedings of the National Academy of Sciences of the United States, 36, 48-49. http://dx.doi.org/10.1073/pnas.36.1.48

[4] Landsburg, S. (2011) Nash Equilibria in Quantum Games. Proceedings of the American Mathematical Society, 139, 4423-4434. http://dx.doi.org/10.1090/S0002-9939-2011-10838-4

[5] Ahmed, A. (2013) Quantum Games and Quaternionic Strategies. Quantum Information Processing, 12, $2701-2720$. http://dx.doi.org/10.1007/s11128-013-0553-5

[6] Ahmed, A. (2013) Equilibria in Quantum Three-Player Dilemma Game. British Journal of Mathematics \& Computer Science, 3, 195-208. http://dx.doi.org/10.1007/s11128-013-0553-5

[7] Baez, J. (2002) The Octonions. Bulletin of the American Mathematical Society, 39, 145-205. http://dx.doi.org/10.1090/S0273-0979-01-00934-X

[8] Conway, J.H. and Smith, D.A. (2003) On Quaternions and Octonions. A. K. Peters, Wellesley, Massachusetts.

[9] Ahmed, A. (2011) On Quaternions, Octonions, and the Quantization of Games: A Text on Quantum Games. Lambert 
A. Shaik, A. Ahmed

Academic Publishing, Saarbrücken.

[10] Ahmed, A.O., Bleiler, S.A. and Khan, F.S. (2010) Octonionization of Three-Player, Two-Strategy Maximally Entangled Quantum Games. International Journal of Quantum Information, 8, 411-434.

http://dx.doi.org/10.1142/S0219749910006344 
Scientific Research Publishing (SCIRP) is one of the largest Open Access journal publishers. It is currently publishing more than 200 open access, online, peer-reviewed journals covering a wide range of academic disciplines. SCIRP serves the worldwide academic communities and contributes to the progress and application of science with its publication.

Other selected journals from SCIRP are listed as below. Submit your manuscript to us via either submit@scirp.org or Online Submission Portal.
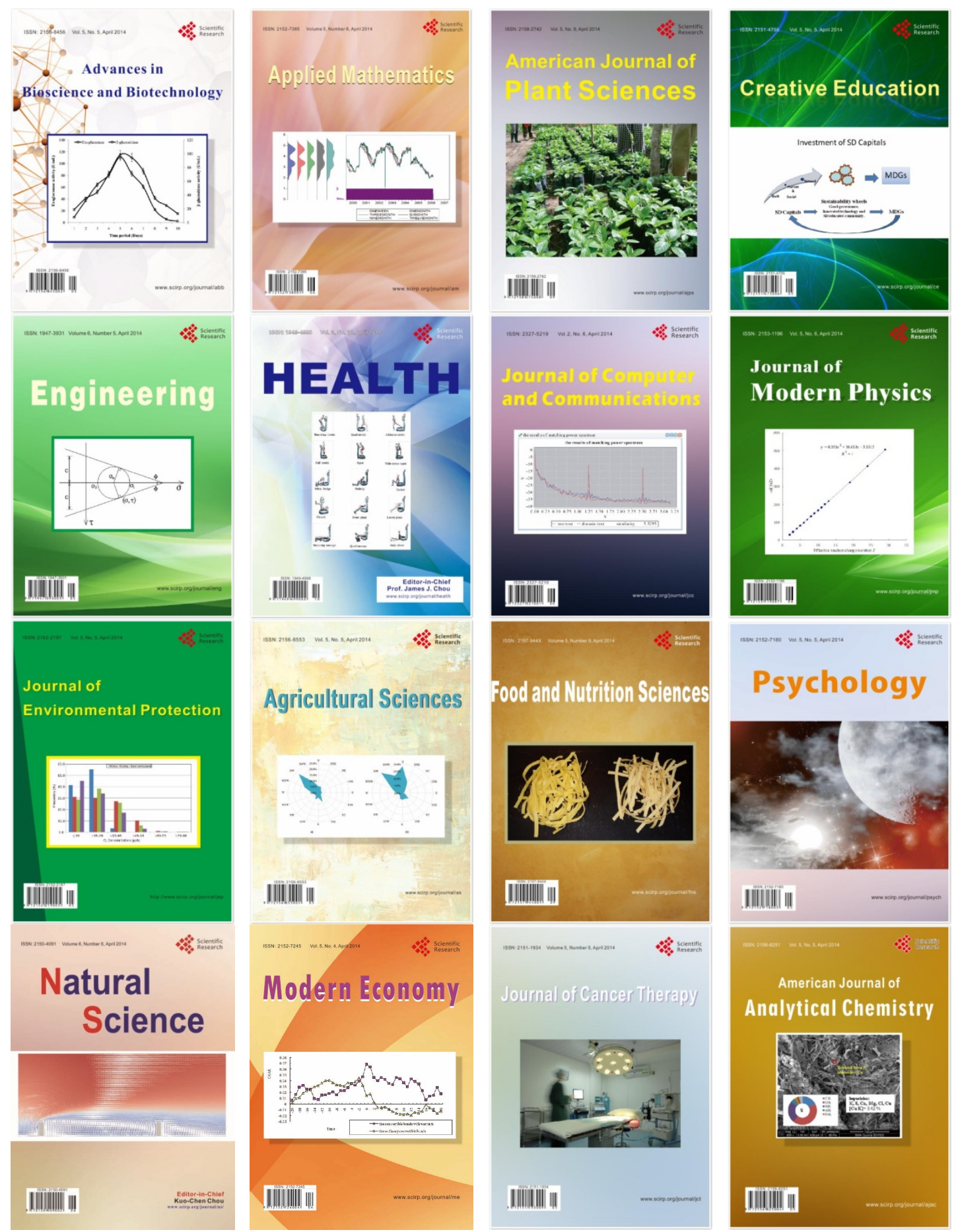